\title{
Daily feeding rhythm linked to microbiome composition in two zooplankton species
}

\author{
Alaina Pfenning-Butterworth $\mathbb{1}^{\circledR *}$, Reilly O. Cooper ${ }^{\circledR}$, Clayton E. Cressler \\ School of Biological Sciences, University of Nebraska-Lincoln, Lincoln, Nebraska, United States of America \\ จ These authors contributed equally to this work. \\ *apfenning@ @uskers.unl.edu
}

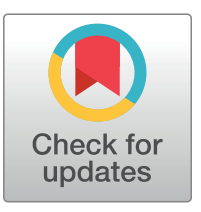

\section{OPEnACCESS}

Citation: Pfenning-Butterworth A, Cooper R0, Cressler CE (2022) Daily feeding rhythm linked to microbiome composition in two zooplankton species. PLoS ONE 17(2): e0263538. https://doi. org/10.1371/journal.pone.0263538

Editor: Juan J Loor, University of Illinois, UNITED STATES

Received: November 15, 2021

Accepted: January 20, 2022

Published: February 3, 2022

Peer Review History: PLOS recognizes the benefits of transparency in the peer review process; therefore, we enable the publication of all of the content of peer review and author responses alongside final, published articles. The editorial history of this article is available here: https://doi.org/10.1371/journal.pone.0263538

Copyright: @ 2022 Pfenning-Butterworth et al. This is an open access article distributed under the terms of the Creative Commons Attribution License, which permits unrestricted use, distribution, and reproduction in any medium, provided the original author and source are credited.

Data Availability Statement: Raw reads for the Daphnia magna and $D$. dentifera samples are available on NCBI (BioProject PRJNA715454). All code used to generate statistics and figures as well

\section{Abstract}

Host-associated microbial communities are impacted by external and within-host factors, i.e., diet and feeding behavior. For organisms known to have a circadian rhythm in feeding behavior, microbiome composition is likely impacted by the different rates of microbe introduction and removal across a daily cycle, in addition to any diet-induced changes in microbial interactions. Here, we measured feeding behavior and used 16S rRNA sequencing to compare the microbial community across a diel cycle in two distantly related species of Daphnia, that differ in their life history traits, to assess how daily feeding patterns impact microbiome composition. We find that Daphnia species reared under similar laboratory conditions have significantly different microbial communities. Additionally, we reveal that Daphnia have daily differences in their microbial composition that correspond with feeding behavior, such that there is greater microbiome diversity at night during the host's active feeding phase. These results highlight that zooplankton microbiomes are relatively distinct and are likely influenced by host phylogeny.

\section{Introduction}

The structure and function of host-associated microbial communities are linked with both intrinsic host factors and external factors. In particular, diet quality and variety influence microbiome composition; for example, mice transitioned from wild diets to controlled laboratory diets experienced shifts in microbiome composition and microbial functional pathways associated with carbohydrate metabolism, sugar metabolism, and motility [1]. Consequently, these compositional and functional changes correlate with changes in host behavior [2], host immune function [3], and other physiological functions [4].

Host diet is strongly influenced by feeding behavior, and many organisms show a daily rhythm in feeding behavior that is regulated by clock genes [5]. This rhythm could impact microbiome composition, both by altering diet-mediated microbial interactions and by modifying the intake of new microbes through feeding and the removal of existing microbes through peristalsis. However, there are few studies that link changes in feeding behavior and microbial composition, despite the downstream effects changes in the microbiome can have on host survival, fitness, and immunity [6].

To understand the links between biological rhythms, feeding behavior, and microbiome composition, we use the Daphnia model system. Daphnia are dominant herbivores and serve 
as raw data are available on GitHub (https://github. com/reillyowencooper/circadian_microbiome).

Funding: The author(s) received no specific funding for this work.

Competing interests: The authors have declared that no competing interests exist. as keystone species by linking primary producers with secondary consumers in freshwater systems [7]. Daphnia are an ideal system for addressing if and how feeding behavior and microbial composition are linked because (1) they have a relatively simple microbiome, which has been characterized in multiple species [8,9], (2) they have core clock genes and show circadian expression of feeding behavior, immune, and sensory genes [10-13], and (3) quantifying individual feeding behavior is straightforward [14].

We examined two species of Daphnia, D. dentifera and D. magna. These species are not phylogenetically closely related, and they exhibit differences in life history traits $[15,16]$ that likely affect host-microbiome interactions. For instance, diel vertical migration (DVM), where Daphnia migrate to the top of the water column at night to feed [17], is thought to be an ancestral trait of all Daphnia species. However, more recent studies suggest that some species exhibit DVM more consistently than others. In D. magna DVM may be tightly linked to genotype [18, 19]; whereas $D$. dentifera show a robust DVM behavior in laboratory and natural systems regardless of genotype [20]. Daphnia dentifera also have a circadian rhythm in feeding behavior that is consistent with DVM [10]. Given these differences, we predicted that D. dentifera's microbiome composition would be more diverse during their active phase (night). In contrast, we predicted that $D$. magna would not have differences in their microbiome composition across a day given that they are not known to exhibit a strong DVM.

We used $16 \mathrm{~S}$ rRNA sequencing to compare the microbiome composition of individually reared Daphnia of each species to their feeding behavior across a diel cycle to address the following questions: (1) Are there microbiome composition differences among species of Daphnia? (2) Is there time of day differences in microbiome composition within each species and are the differences consistent with feeding behavior? (3) If there are daily differences in microbiome composition, are they consistent across both host species?

We found that $D$. dentifera and $D$. magna have significantly different microbiome composition. Additionally, microbiome composition does vary with time of day and is consistent with their feeding behavior, such that increased feeding and higher microbiome diversity occurred at night in both host species. However, there are host-specific differences in the abundance of specific microbes with time of day, suggesting that host feeding behavior and microbial community abundance are linked.

\section{Materials and methods}

\section{Experimental design}

Both Daphnia dentifera and D. magna were maintained in cultures for more than 2 and 5 years, respectively. Cultured individuals were maintained at $22^{\circ} \mathrm{C}$, in COMBO medium [21], under a 15:9 light:dark cycle and were fed batch-cultured Ankistrodesmus falcatus in $500 \mathrm{ml}$ of high nitrogen $\mathrm{COMBO}$ under constant light at $22^{\circ} \mathrm{C}$. Individuals were moved to new COMBO and fed $A$. falcatus every other day at quantities relative to their body size $(1 \mathrm{mgC} / \mathrm{L}$ for $D$. dentifera and $2.5 \mathrm{mgC} / \mathrm{L}$ for D. magna). At fourteen days old individuals were collected for DNA extraction.

\section{Feeding assay}

Prior to the feeding assay, individual D. dentifera and D. magna were reared to 14 days old as described above with one exception. Individuals were fed 14 day old $A$. falcatus that was frozen in daily aliquots for the course of the feeding rate assay. This ensured that (1) individuals were fed a consistent chlorophyll:carbon ratio and (2) that algal quantity remained the same during feeding rate assays run in the dark versus in the light. Individual $D$. dentifera and D. magna were randomly assigned to either the day or night feeding rate assay $(n=14, N=56)$. 
To quantify feeding rate, we isolated D. dentifera and D. magna individuals in $15 \mathrm{ml}$ and 30 $\mathrm{ml}$ tubes (Nunc, Rochester, NY, USA) with 10 and $30 \mathrm{~mL}$ of COMBO media combined with $1 \mathrm{mgC/L}$ of $A$. falcatus, respectively. Individuals fed for six hours, after which they were moved to a new vial with fresh $\mathrm{COMBO}$ and their body size was measured. We measured algal fluorescence on a plate reader (Tecan, Maennedorf, Switzerland) following Hite et al. 2020, to compare the relative quantities of chlorophyll- $a$ in wells with animals to control wells that did not contain animals [14]. We calculated individual feeding rates as:

$$
\text { Feeding rate }=\ln \left(\mathrm{F}_{\text {control }} / \mathrm{F}_{\text {Daphnia }}\right) * \mathrm{v} / \mathrm{t}
$$

where $\mathrm{F}_{\text {control }}$ is the average of fluorescence of control wells, $\mathrm{F}_{\text {Daphnia }}$ is the fluorescence of an animal well, $\mathrm{v}$ is the volume of COMBO and algae in $\mathrm{ml}$, and $\mathrm{t}$ is the time individuals fed in hours [22]. We corrected each feeding rate by individual body size to account for species specific differences in feeding rate.

\section{DNA extraction and sequencing}

Daphnia magna were pooled in sets of 5 individuals $(\mathrm{n}=10$ samples per time point) and $D$. dentifera in sets of 10 individuals ( $\mathrm{n}=10$ samples per time point) for DNA extraction. DNA was extracted using the DNEasy Blood \& Tissue Kit, with a 24 h proteinase K digestion step to ensure that all microbial cells within and on hosts were digested. The 16S rRNA V4 hypervariable region was amplified using the 515f/806r primer pair [23] and PCR steps of $95^{\circ} \mathrm{C}$ for 3 min; 35 cycles of $95^{\circ} \mathrm{C}$ for $45 \mathrm{sec}, 58^{\circ} \mathrm{C}$ for $30 \mathrm{sec}, 72^{\circ} \mathrm{C}$ for $45 \mathrm{sec}$; and $72^{\circ} \mathrm{C}$ for 5 minutes. Sample libraries were normalized with the SequalPrep Normalization Plate Kit, then quality checked using the KAPA Library Quantification Kit. Samples were pooled and PhiX spiked, then sequenced using the MiSeq Reagent Kit v2 (300 cycles) on the Illumina MiSeq (Nebraska Food for Health Center, Lincoln, NE, USA).

\section{Sequence analysis, visualization, and statistics}

Demultiplexed samples were processed using the dada2 v1.16.0 [24] algorithm in R [25]. After read processing, amplicon sequence variants (ASVs) were assigned taxonomy using the dada2-formatted GTDB taxonomy reference database v3 [26]. Sample metadata, processed ASVs, taxonomic identity, and a phylogenetic tree constructed using the phangorn v2.5.5 [27] and DECIPHER v2.16.1 [28] packages were combined in a phyloseq v1.32.0 [29] object for downstream visualization and statistics. To analyze alpha diversity, the inverse Simpson index was estimated for each sample using DivNet v0.3.6 [30]. An ANOVA was used to analyze alpha diversity differences across host species and time points, then posthoc Tukey honest significant differences were calculated to understand differences among treatments. Beta diversity was measured across samples using weighted UniFrac distance, and statistically analyzed using PERMANOVAs across host species and time points. Core taxa in the microbiota of both species were identified using the microbiome package v1.10.0 [31], with taxa appearing across at least $50 \%$ of samples and at relative abundances $\geq 0.001$ included in the analysis. To find taxa that differed significantly between time points, we focused on relatively common ASVs ( $\geq 0.005$ relative abundance) and compared dayversus-night relative abundance using two statistical tests. For ASVs that were unique to one host species, we compared the relative abundance of each ASV at day versus night using a t-test. For ASVs abundant in both host species we compared the relative abundances of each ASV at day versus night using ANOVA. 


\section{Results}

\section{Composition of the microbiota is different across host species}

Though both Daphnia dentifera and D. magna are freshwater zooplankton species and their rearing conditions are matched, their microbiomes differ substantially (Fig 1). We found that

a

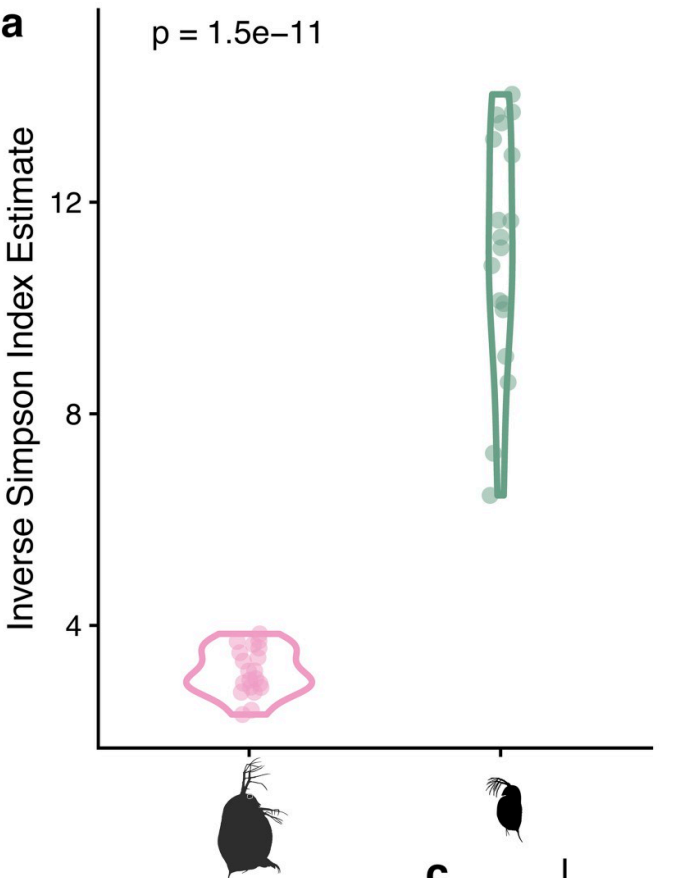

C

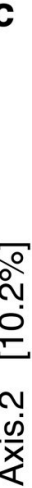

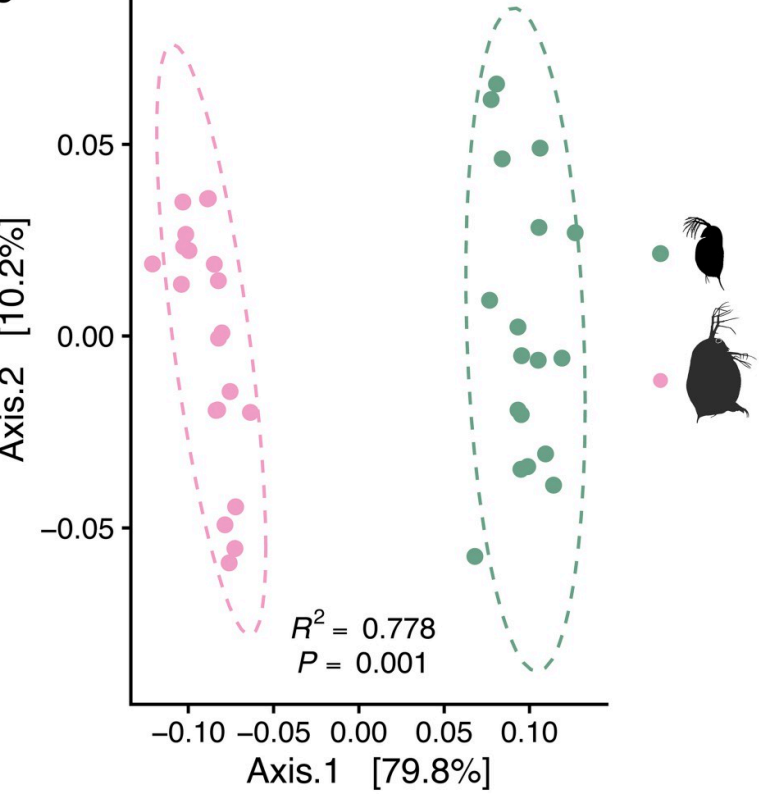

b
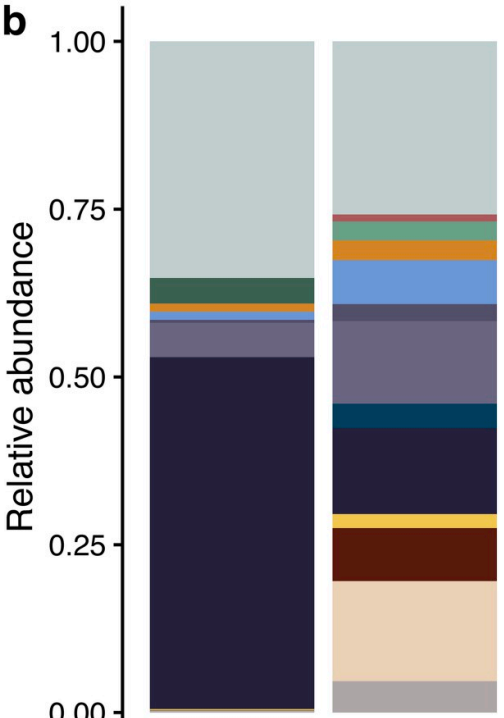

Genus

$\leq 1 \%$ Abundant/Unidentified

Acidovorax_D

Arcticibacterium

BRH-c54

Emticicia

Flavobacterium

Fluviicola

Hydromonas

Hylemonella

Limnohabitans

Nevskia

Planktophila

Polynucleobacter

Vitreoscilla_A

0.00

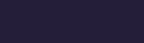

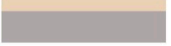
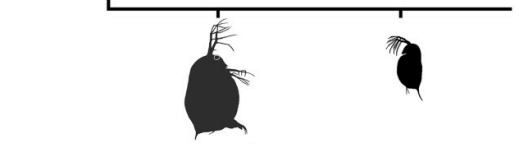

Species \& Time Legend

Fig 1. Host species differences in microbiota structure. a) Inverse Simpson index estimates for individual samples of Daphnia magna and D. dentifera, with t-test results reported. b) Genus-level bacterial microbiota composition for D. magna and D. dentifera. Amplicon sequence variants (ASVs) identified at $<=1 \%$ of relative abundance in each host species and ASVs not able to be identified at the Genus level are denoted by " $<=1 \%$ Abundant/Unidentified". c) Principal coordinate analysis using weighted UniFrac distances comparing D. magna and D. dentifera, with PERMANOVA results reported. Ellipses represent the multivariate t-distribution of D. magna and D. dentifera samples. 
D. dentifera harbor a significantly more diverse set of bacterial taxa than D. magna (Fig 1a, Inverse Simpson index, D. dentifera $=11.39 \pm 2.62, \mathrm{n}=19, D$. magna $=3.13 \pm 0.44, \mathrm{n}=20$, $p<0.0001$ ). The $D$. dentifera microbiome is not dominated by a single genus; instead, many genera exist at low abundances (Fig 1b). In contrast, the D. magna microbiome is dominated by the Limnohabitans genus ( $>50 \%$ relative abundance) and lacks many of the genera identified in the $D$. dentifera microbiome. In total, we find that community composition within each host species clusters together, indicating that $D$. magna and $D$. dentifera have statistically distinct bacterial communities (Fig 1c, PERMANOVA, pseudo- $F_{1,37}=124.47, \mathrm{R}^{2}=0.778, p=0.001$ ).

\section{Composition of the microbiota and feeding behavior varies across a day}

Between day and night, we observed changes in both feeding behavior and microbial community diversity and composition in both Daphnia dentifera and D. magna (Fig 2). Both host species had significantly more diverse microbiomes at night than during the day (Fig 2a, $D$. magna: t-test $p<0.0001, D$. dentifera: t-test $p=0.004$ ). In addition, systematic shifts in composition between day and night were observed in both species (Fig 2c). In D. magna, increased bacterial diversity at night corresponded with an increase in the relative abundance of Arcticibacterium, a decrease in Limnohabitans, and a large increase in the relative abundance of rare taxa each appearing at less than $5 \%$ abundance (increasing in total from a maximum relative abundance of $35 \%$ to a maximum of 55\%), though no rare taxa became prominent enough to surpass any of the taxa consistently present at $>5 \%$ relative abundance. Daphnia dentifera composition changes were more difficult to disentangle, but the more diverse night microbiome corresponded with lower abundances of Hydromonas and an increase in the relative abundances of rare taxa. We also found that both Daphnia species had significantly higher feeding rates during the night than the day (Fig 2b, D. magna: paired t-test $p=0.008, D$. dentifera: paired t-test $p<0.0001$ ).

\section{Specific amplicon sequence variants are associated with shifts in community composition across a day}

To investigate the shifting microbiome composition in both host species beyond the genus taxonomic rank, we identified ASVs with relative abundances greater than $.05 \%$ found only in $D$. dentifera, D. magna, or shared between the two species. We then examined whether relative abundance of these identified taxa differed during the day or night (Fig 3). Simultaneously, we identified ASVs that were core members of the microbiome in each species (S1 Table). We found seven ASVs that were considered core members in both host species, all belonging to the Gammaproteobacteria or Bacteroidia. We found a further 13 core ASVs in D. magna and 27 core ASVs in D. dentifera, though many of these ASVs were found at relative abundances less than $0.05 \%$.

In ASVs specific to D. magna, only three experienced significant shifts between day and night: an Arcticibacterium ( $p=0.013)$, Emticicia ASV $1(p=0.038)$, and a Polyangiaceae $(p=0.004)$ were more relatively abundant at night (Fig 3a). More unique ASVs were found in $D$. dentifera, and more were significantly different between time points: a Polynucleobacter ( $p=0.001)$ and Lacibacter $(p=0.047)$ were more relatively abundant during the day, while Limnohabitans ASV 1 ( $p=0.017)$, an NS11-12g $(p=0.032)$, and Limnobacter $(p=0.001)$ were more relatively abundant at night (Fig $3 \mathrm{~b}$ ). For ASVs found in both species (Fig $3 \mathrm{c}$ and $3 \mathrm{~d}$ ), only Limnohabitans ASV 3 was significantly different at the time points in both species. However, the abundance shift was opposite in the two species; in D. magna, Limnohabitans ASV 3 was more abundant during the day $(p<0.0001)$, but in $D$. dentifera it was more abundant at night ( $p=0.005)$. In D. magna, a Flavobacterium ASV was more abundant at night $(p=0.002)$. The same Flavobacterium was more abundant during the night in D. dentifera, though not 
a
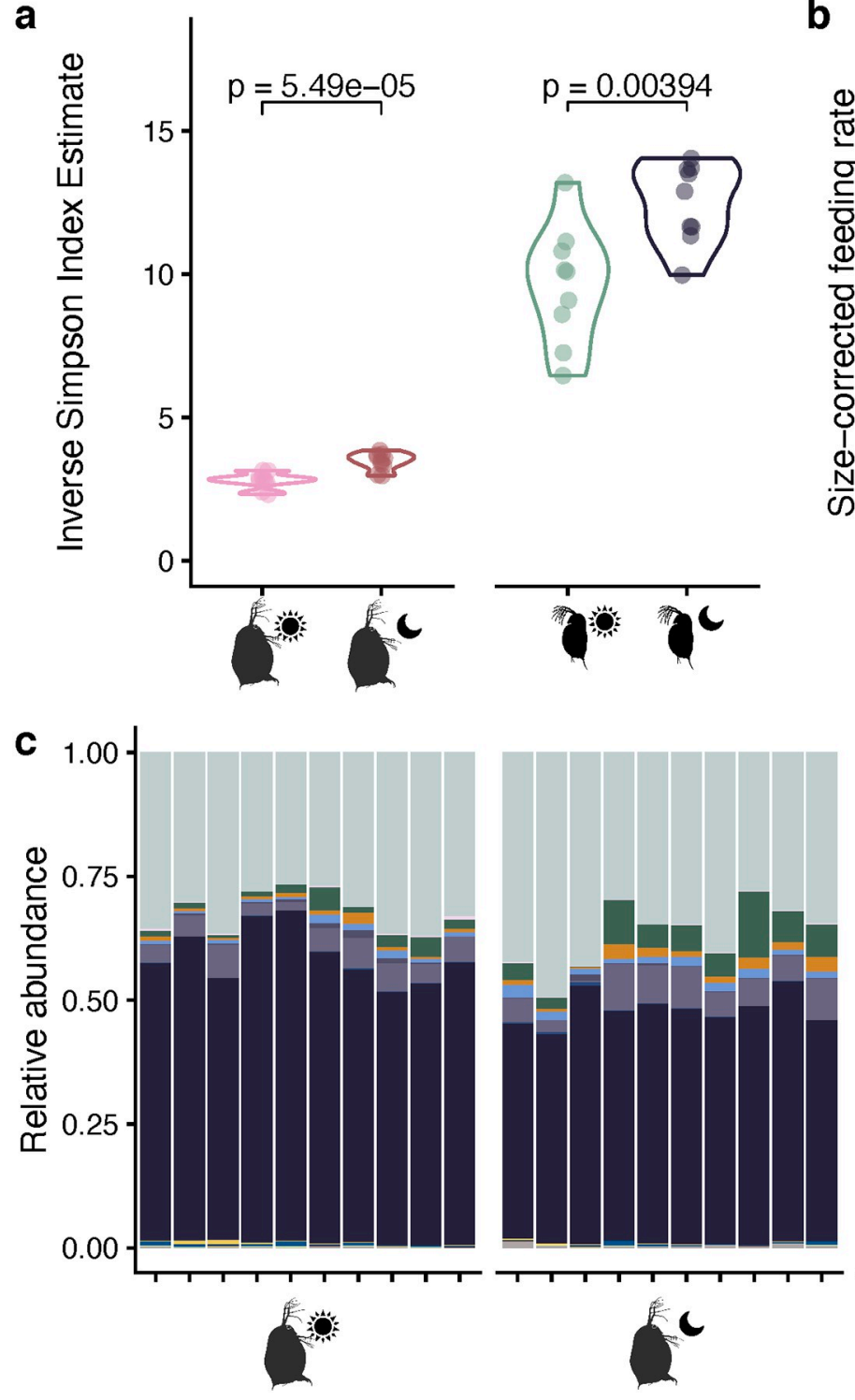

Genus $<=5 \%$ Abundant/Unidentified
Acidovorax_D Aquabacterium

Arcticibacterium

Cypionkella

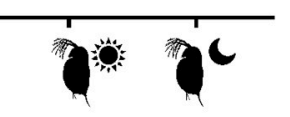

b
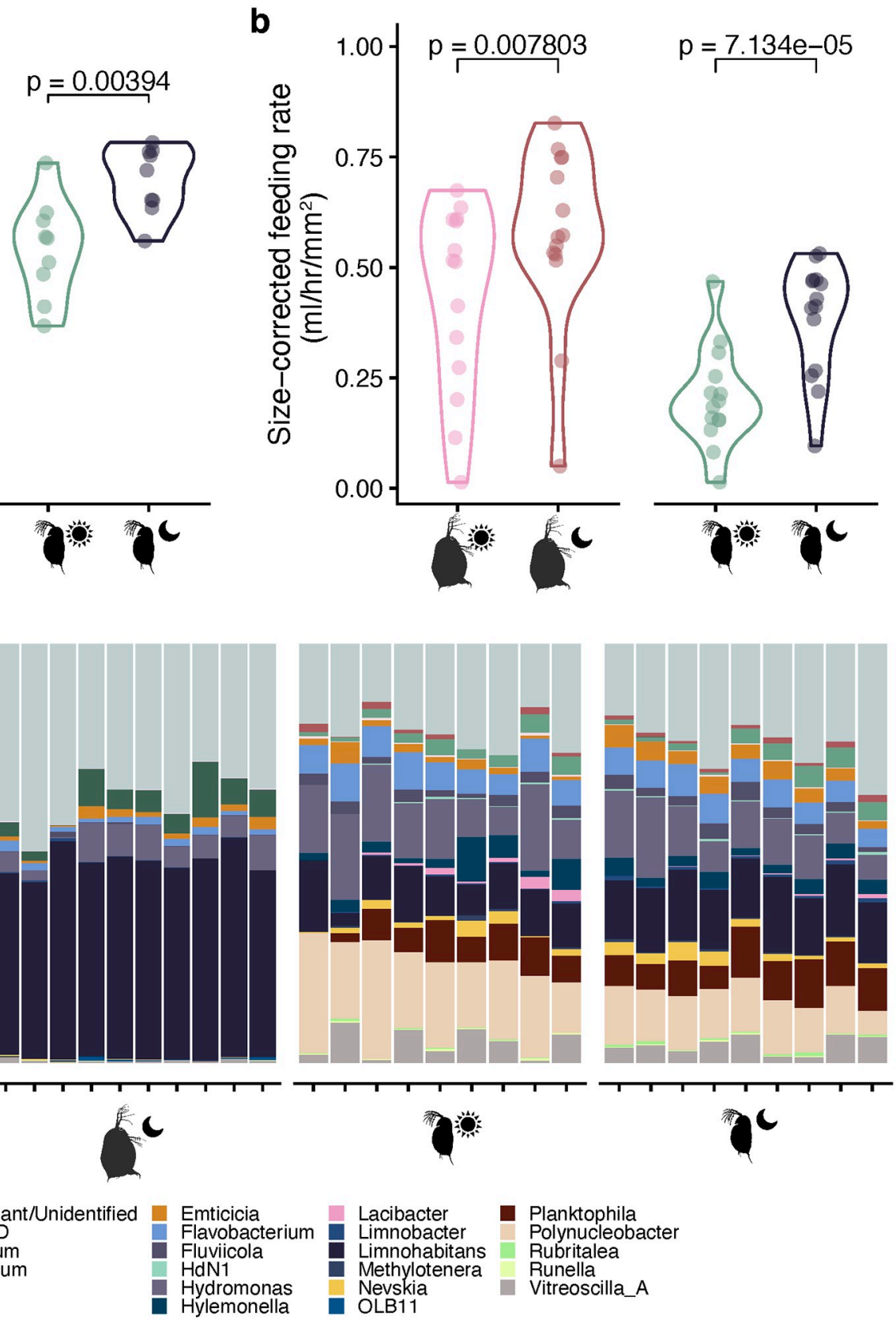

Planktophila Polynucleobacter Rubritalea Runella

Vitreoscilla A

Fig 2. Differences in microbiota structure at different times of day. (a) Alpha diversity, represented by the inverse Simpson index, of Daphnia magna and $D$. dentifera during the day and night sample timepoints. For each species t-test results are reported. (b) Size-corrected feeding rates of $D$. magna and $D$. dentifera during the day and night sample time points $(\mathrm{n}=14$ per species). For each species t-test results are reported. (c) Genus-level composition plots for each sample taken for D. magna and D. dentifera at each timepoint (D. magna, $\mathrm{n}=10 ;$ D. dentifera, $\mathrm{n}=9$ ).

https://doi.org/10.1371/journal.pone.0263538.g002

significantly so $(p=0.06)$. In $D$. dentifera, a Chitinophagaceae ASV $(p=0.002)$ and Emticicia ASV $2(p=0.005)$ were more abundant at night.

\section{Discussion}

Our results show that the microbiomes of Daphnia dentifera and D. magna are distinct. While most host-associated microbiomes are strongly associated with host phylogeny [32-36], the 


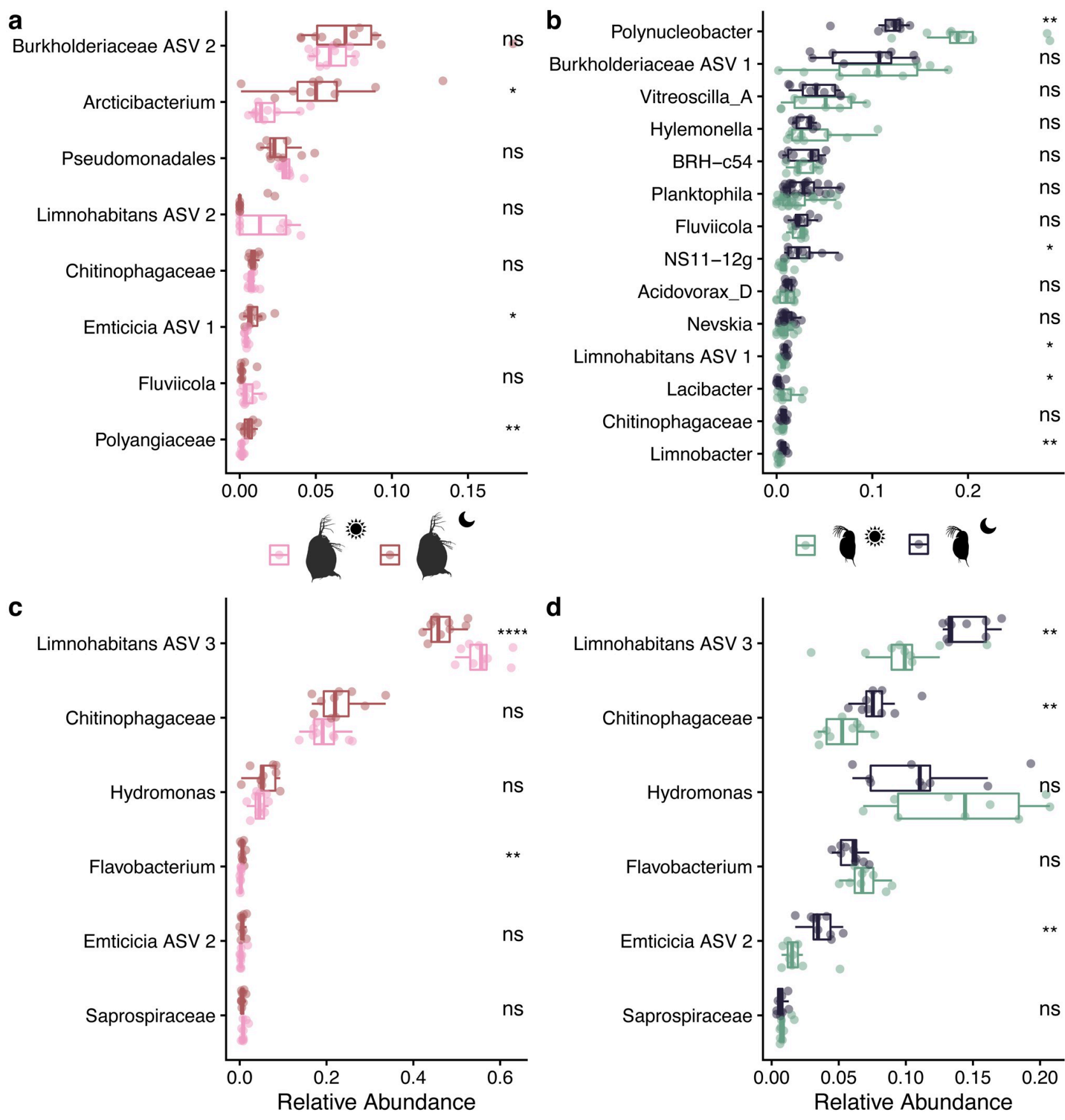

Fig 3. Comparisons of ASVs with more than $\mathbf{0 . 5 \%}$ abundance across all samples by host species and time point. Significance of t-tests for each ASV are denoted on the right side of each figure $\left({ }^{*}, p<0.05 ;{ }^{* *}, p<0.01 ;{ }^{* * *}, p<0.001 ;{ }^{* * * *}, p<0.0001\right.$ ). (a) ASVs found only in Daphnia magna in day (pink) or night (red) samples. ASV taxonomic identity is denoted at the most specific taxonomic rank possible without assigning species-level identity. (b) ASVs found only in D. dentifera in day (green) or night (blue) samples, with taxonomic identity as in (a). (c) \& (d) ASVs common to both host species. Relative abundances of ASVs in D. magna in (c) and relative abundances of ASVs in D. dentifera in (d), with taxonomic identity as in (a).

https://doi.org/10.1371/journal.pone.0263538.g003

differences between these two species are striking, as these two distantly related species have been cultured in similar in-laboratory rearing conditions for years. While phylosymbiosis, the hypothesis that host-associated microbial communities of closely related host species are more similar to each other than to less related host species [37], has been observed in other 
invertebrate species, this pattern is not frequently observed in zooplankton species, as much of their microbiome is acquired from the environment $[8,38]$. The differences in community composition between D. dentifera and D. magna in this study, as well as the differences among other zooplankton species as demonstrated by Eckert et al. [8], provide some evidence that zooplankton microbiomes are relatively distinct. However, our results indicated that microbiome composition are not a result of environmental conditions.

Here, we find that D. magna has a far less diverse microbiome than $D$. dentifera. The microbiome of D. magna is consistently dominated by a Limnohabitans ASV and a Chitinophagaceae ASV, while the microbiome of $D$. dentifera is evenly distributed across several Burkholderiaceae ASVs (including Limnohabitans, Hydromonas, and other unidentified Burkholderiacae). Though both Daphnia species were reared in similar laboratory conditions their microbiomes were dramatically different. The Daphnia dentifera used here were originally collected from temperate lakes in Southern Michigan (USA) and the D. magna are from a pond at Kaimes Farm, Leitholm, Scottish Borders [39]. The microbiome composition differences observed may be remnants of the microbes in their natural habitat, despite having been reared in the lab for hundreds of generations. In concert with evidence that zooplankton have distinct microbiomes as compared to their local environment $[9,40,41]$, these results suggest that these host species can actively shape and maintain their associated microbial communities.

Host behavior and the microbiome are intrinsically linked [42-44]. While the microbiomes of these zooplankton species are clearly distinct, the same patterns of increased bacterial diversity and increased feeding rate at night emerge here. This suggests that microbiome diversity is linked to feeding behavior in D. dentifera and D. magna, and likely both are tied to strong diel vertical migration behaviors present across Daphnia species [45-47]. Increased feeding rate at night may influence microbiome composition and diversity through dietary changes; across host species, diet is strongly associated with microbiome structure [4, 48-50]. Diet is also directly associated with host behavior, as both quality and quantity of available food influences how hosts forage [51-53]. Because diet can impact microbiome structure and composition, it is also likely that diet-derived nutrients taken up by taxa in the microbiome indirectly impact host behavior [54] and that there is a strong link between feeding behavior and the microbiome. Demonstrated microbiome-mediated behavioral changes include those involved in stressrelated behavior [55] and social behavior [42, 56]. In Daphnia species, diel vertical migration (DVM) is a host behavior intrinsically tied to feeding. Zooplankton consume more food at night when migrating up the water column in the relative safety of darkness from visual predators, then return to darker, deeper layers of the water column in daylight, where they consume less food [10,57]. Because Daphnia in this experiment were raised individually in $30 \mathrm{~mL}$ tubes, we demonstrate that changes in feeding behavior observed here are strongly associated with changes in microbial diversity in the absence of host migration through the water column.

Though it is difficult to infer the functional importance of taxa in the microbiota, prior work characterizing metagenome-assembled genomes of Daphnia magna has pinpointed some functions encoded by their microbes. In particular, two Limnohabitans species and one Emticicia species have had some functions characterized [58], which are likely the same species as the ASVs identified as Limnohabitans ASVs (Limnohabitans ASV 2 and 3, Fig 3) and the Emticica ASV (Emticicia ASV 2, Fig 3) in this study. Many of the functions encoded in these genomes involve amino acid biosynthesis and transport, vitamin and mineral transport, and carbohydrate degradation, and many pathways encoded by the whole $D$. magna metagenome are implicated in carbohydrate breakdown. This suggests that many of the taxa in the Daphnia microbiota are involved in sequestering usable nutrients from carbohydrates taken in while Daphnia feed. 
In conclusion, our results suggest that zooplankton hosts may be able to maintain microbial communities associated with their natural environment even with substantial pressure of the current environment on zooplankton microbiome composition [8]. This work also emphasizes the link between daily rhythms in behavior, specifically feeding, and the microbiome. Incorporating metabolomics to infer functional differences could provide further insight into the host's metabolic processes. The continued pursuit to understand how and when daily rhythms in behavior and the microbiome impact within-host processes could provide key insights into metabolic and immune disorders.

\section{Supporting information}

S1 Table. ASVs that are core members of the microbiome in each host species. The mean relative abundance in both host species is listed for each ASV as well as the taxonomic identity. (PDF)

\section{Acknowledgments}

We thank Dr. Andrew Benson, Mallory Van Haute, and Qinnan Yang for their assistance with library preparation and amplicon sequencing. We credit Mathilde Cordellier for the Daphnia magna image used in figures.

\section{Author Contributions}

Conceptualization: Alaina Pfenning-Butterworth, Reilly O. Cooper.

Data curation: Alaina Pfenning-Butterworth, Reilly O. Cooper.

Formal analysis: Alaina Pfenning-Butterworth, Reilly O. Cooper.

Writing - original draft: Alaina Pfenning-Butterworth, Reilly O. Cooper.

Writing - review \& editing: Alaina Pfenning-Butterworth, Reilly O. Cooper, Clayton E. Cressler.

\section{References}

1. Wang J, Linnenbrink M, Künzel S, Fernandes R, Nadeau MJ, Rosenstiel P, et al. Dietary history contributes to enterotype-like clustering and functional metagenomic content in the intestinal microbiome of wild mice. PNAS. 2014; 111(26):e2703-10. https://doi.org/10.1073/pnas.1402342111 PMID: 24912178

2. Najarro MA, Sumethasorn M, Lamoureux A, Turner TL. Choosing mates based on the diet of your ancestors: replication of non-genetic assortative mating in Drosophila melanogaster. PeerJ. 2015; 3: e1173. https://doi.org/10.7717/peerj.1173 PMID: 26339551

3. Udayangani RM, Dananjaya SH, Nikapitiya C, Heo GJ, Lee J, De Zoysa M. Metagenomics analysis of gut microbiota and immune modulation in zebrafish (Danio rerio) fed chitosan silver nanocomposites. Fish \& shellfish immunology. 2017; 66:173-84. https://doi.org/10.1016/j.fsi.2017.05.018 PMID: 28479399

4. Singh RK, Chang HW, Yan DI, Lee KM, Ucmak D, Wong K, et al. Influence of diet on the gut microbiome and implications for human health. Journal of translational medicine. 2017; 15(1):1-7.

5. Schibler U, Ripperger J, Brown SA. Peripheral circadian oscillators in mammals: time and food. Journal of biological rhythms. 2003; 18(3):250-60. https://doi.org/10.1177/0748730403018003007 PMID: 12828282

6. Zheng D, Ratiner K, Elinav E. Circadian influences of diet on the microbiome and immunity. Trends in immunology. 2020; 41(6):512-30. https://doi.org/10.1016/j.it.2020.04.005 PMID: 32359722

7. Sarnelle O. Daphnia as keystone predators: effects on phytoplankton diversity and grazing resistance. Journal of plankton research. 2005; 27(12):1229-38. 
8. Eckert EM, Anicic N, Fontaneto $D$. Freshwater zooplankton microbiome composition is highly flexible and strongly influenced by the environment. Molecular Ecology. 2021; 30(6):1545-58. https://doi.org/ 10.1111/mec.15815 PMID: 33484584

9. Freese HM, Schink B. Composition and stability of the microbial community inside the digestive tract of the aquatic crustacean Daphnia magna. Microbial ecology. 2011; 62(4):882. https://doi.org/10.1007/ s00248-011-9886-8 PMID: 21667195

10. Pfenning-Butterworth AC, Amato K, Cressler CE. Circadian rhythm in feeding behavior of Daphnia dentifera. Journal of Biological Rhythms. 2021; 36(6):589-594. https://doi.org/10.1177/ 07487304211054404 PMID: 34753340

11. Cellier-Michel S, Rehaïlia M, Berthon JL. Is the rhythm of vertical migration of Daphnia longispina circadian or simply nycthemeral?. Annales de Limnologie-International Journal of Limnology 2003; 39 (3):265-272.

12. Rund SS, Yoo B, Alam C, Green T, Stephens MT, Zeng E, et al. Genome-wide profiling of $24 \mathrm{hr}$ diel rhythmicity in the water flea, Daphnia pulex. network analysis reveals rhythmic gene expression and enhances functional gene annotation. BMC genomics. 2016; 17(1):1-20. https://doi.org/10.1186/ s12864-016-2998-2 PMID: 27538446

13. Schwarzenberger A, Chen L, Weiss LC. The expression of circadian clock genes in Daphnia magna diapause. Scientific reports. 2020; 10(1):1-7.

14. Hite JL, Pfenning-Butterworth AC, Vetter RE, Cressler CE. A high-throughput method to quantify feeding rates in aquatic organisms: A case study with Daphnia. Ecology and evolution. 2020; 10(13):623945. https://doi.org/10.1002/ece3.6352 PMID: 32724510

15. Zuykova El, Bochkarev NA, Sheveleva NG. Genetic polymorphism, haplotype distribution, and phylogeny of Daphnia (Cladocera: Anomopoda) species from the water bodies of Russia as inferred from the $16 S$ mtDNA gene sequencing. Russian journal of genetics. 2016; 52(6):585-96.

16. Adamowicz SJ, Petrusek A, Colbourne JK, Hebert PD, Witt JD. The scale of divergence: a phylogenetic appraisal of intercontinental allopatric speciation in a passively dispersed freshwater zooplankton genus. Molecular phylogenetics and evolution. 2009; 50(3):423-36. https://doi.org/10.1016/j.ympev. 2008.11.026 PMID: 19124080

17. Dawidowicz P, Pijanowska J. Diel vertical migration of aquatic crustaceans_-adaptive role, underlying mechanisms, and ecosystem consequences. The Natural History of the Crustacea: Life Histories. 2018 May 22:231-56.

18. De Meester L. An analysis of the phototactic behaviour of Daphnia magna clones and their sexual descendants. In Biology of Cladocera 1991 (pp. 217-227). Springer, Dordrecht.

19. De Meester L. Fish-mediated chemical, and phototactic behaviour in Daphnia magna. Ecology. 1993; 74:1467-74.

20. Stich HB. Spatial and temporal heterogeneity of Daphnia in Lake Constance; intra-and interspecific comparisons. Limnology and Oceanography. 1992; 37(6):1327-34.

21. Kilham SS, Kreeger DA, Lynn SG, Goulden CE, Herrera L. COMBO: a defined freshwater culture medium for algae and zooplankton. Hydrobiologia. 1998; 377(1):147-59.

22. Sarnelle O, Wilson AE. Type III functional response in Daphnia. Ecology. 2008; 89(6):1723-32. https:// doi.org/10.1890/07-0935.1 PMID: 18589536

23. Caporaso JG, Lauber CL, Walters WA, Berg-Lyons D, Lozupone CA, Turnbaugh PJ, et al. Global patterns of 16S rRNA diversity at a depth of millions of sequences per sample. PNAS. 2011; 108(Supplement 1):4516-4522.

24. Callahan BJ, McMurdie PJ, Rosen MJ, Han AW, Johnson AJ, Holmes SP. DADA2: high-resolution sample inference from Illumina amplicon data. Nature methods. 2016; 13(7):581-3. https://doi.org/10. 1038/nmeth.3869 PMID: 27214047

25. R Core Team. R: A language and environment for statistical computing. R Foundation for Statistical Computing, Vienna, Austria. 2020; https://www.R-project.org/.

26. Alishum A. DADA2 formatted 16S rRNA gene sequences for both bacteria \& archaea. Zenodo. 2019; https://doi.org/10.5281/zenodo.3266798

27. Schliep K. phangorn: phylogenetic analysis in R. Bioinformatics. 2011; 27(4), 592-593. https://doi.org/ 10.1093/bioinformatics/btq706 PMID: 21169378

28. Wright ES. Using DECIPHER v2.0 to Analyze Big Biological Sequence Data in R. The R Journal. 2016; 8(1), 352-359.

29. McMurdie PJ, Holmes S. phyloseq: An R Package for Reproducible Interactive Analysis and Graphics of Microbiome Census Data. PLoS ONE. 2013; 8(4):e61217. https://doi.org/10.1371/journal.pone. 0061217 PMID: 23630581 
30. Willis AD, Martin BD. Estimating diversity in networked ecological communities. Biostatistics. 2020; 00,1-17.

31. Lahti L, Shetty S, et al. Tools for microbiome analysis in R. Microbiome package version 1.15.1. Bioconductor, 2017; http://microbiome.github.com/microbiome/.

32. Amato KR, Martinez-Mota R, Righini N, Raguet-Schofield M, Corcione FP, Marini E, et al. Phylogenetic and ecological factors impact the gut microbiota of two Neotropical primate species. Oecologia. 2016; 180(3):717-33. https://doi.org/10.1007/s00442-015-3507-z PMID: 26597549

33. Brooks AW, Kohl KD, Brucker RM, van Opstal EJ, Bordenstein SR. Phylosymbiosis: relationships and functional effects of microbial communities across host evolutionary history. PLoS biology. 2016; 14 (11):e2000225. https://doi.org/10.1371/journal.pbio.2000225 PMID: 27861590

34. Davenport ER, Sanders JG, Song SJ, Amato KR, Clark AG, Knight R. The human microbiome in evolution. BMC biology. 2017; 15(1):1-2.

35. Mazel F, Davis KM, Loudon A, Kwong WK, Groussin M, Parfrey LW. Is host filtering the main driver of phylosymbiosis across the tree of life?. Msystems. 2018; 3(5):e00097-18. https://doi.org/10.1128/ mSystems.00097-18 PMID: 30417109

36. Fitzpatrick CR, Copeland J, Wang PW, Guttman DS, Kotanen PM, Johnson MT. Assembly and ecological function of the root microbiome across angiosperm plant species. PNAS. 2018; 115(6):1157-65. https://doi.org/10.1073/pnas.1717617115 PMID: 29358405

37. Lim SJ, Bordenstein SR. An introduction to phylosymbiosis. Proceedings of the Royal Society B. 2020; 287(1922):20192900. https://doi.org/10.1098/rspb.2019.2900 PMID: 32126958

38. Sison-Mangus MP, Metzger CM, Ebert D. Host genotype-specific microbiota do not influence the susceptibility of $D$. magna to a bacterial pathogen. Scientific reports. 2018; 8(1):1-0.

39. Auld SK, Wilson PJ, Little TJ. Rapid change in parasite infection traits over the course of an epidemic in a wild host-parasite population. Oikos. 2014; 123(2):232-8.

40. Pernthaler J, Rosenberg E, DeLong E, Lory S. Freshwater microbial communities. In Rosenberg, Eugene; DeLong, Edward; Lory, Stefan. The Prokaryotes. Berlin Heidelberg: Springer, 97-112.

41. Mushegian AA, Arbore $R$, Walser JC, Ebert $D$. Environmental sources of bacteria and genetic variation in behavior influence host-associated microbiota. Applied and environmental microbiology. 2019; 85(8): e01547-18. https://doi.org/10.1128/AEM.01547-18 PMID: 30737344

42. Vernier CL, Chin IM, Adu-Oppong B, Krupp JJ, Levine J, Dantas G, et al. The gut microbiome defines social group membership in honey bee colonies. Science advances. 2020; 6(42):eabd3431. https://doi. org/10.1126/sciadv.abd3431 PMID: 33055169

43. Vuong HE, Yano JM, Fung TC, Hsiao EY. The microbiome and host behavior. Annual review of neuroscience. 2017; 40:21-49. https://doi.org/10.1146/annurev-neuro-072116-031347 PMID: 28301775

44. Buffington SA, Dooling SW, Sgritta M, Noecker C, Murillo OD, Felice DF, et al. Dissecting the contribution of host genetics and the microbiome in complex behaviors. Cell. 2021; 184(7):1740-56. https://doi. org/10.1016/j.cell.2021.02.009 PMID: 33705688

45. Haney JF, Hall DJ. Diel vertical migration and filter-feeding activities of Daphnia. Arch. Hydrobiol. 1975 Jan 1; 75(4):413-41.

46. Loose CJ, Dawidowicz P. Trade-offs in diel vertical migration by zooplankton: the costs of predator avoidance. Ecology. 1994; 75(8):2255-63.

47. Ringelberg J. An account of a preliminary mechanistic model of swimming behaviour in Daphnia: its use in understanding diel vertical migration. In Cladocera as Model Organisms in Biology 1995 (pp. 161165). Springer, Dordrecht.

48. David LA, Maurice CF, Carmody RN, Gootenberg DB, Button JE, Wolfe BE, et al. Diet rapidly and reproducibly alters the human gut microbiome. Nature. 2014; 505(7484):559-63. https://doi.org/10.1038/ nature12820 PMID: 24336217

49. Macke E, Callens M, Massol F, Vanoverberghe I, De Meester L, Decaestecker E. Diet and genotype of an aquatic invertebrate affect the composition of free-living microbial communities. Frontiers in microbiology. 2020; 11:380. https://doi.org/10.3389/fmicb.2020.00380 PMID: 32256467

50. Mallott EK, Amato KR, Garber PA, Malhi RS. Influence of fruit and invertebrate consumption on the gut microbiota of wild white-faced capuchins (Cebus capucinus). American journal of physical anthropology. 2018; 165(3):576-88. https://doi.org/10.1002/ajpa.23395 PMID: 29313897

51. Frost BW. Effects of size and concentration of food particles on the feeding behavior of the marine planktonic copepod Calanus pacificus. Limnology and oceanography. 1972; 17(6):805-15.

52. Glander KE. The impact of plant secondary compounds on primate feeding behavior. American Journal of Physical Anthropology. 1982; 25(S3):1-8. 
53. May CE, Vaziri A, Lin YQ, Grushko O, Khabiri M, Wang QP, et al. High dietary sugar reshapes sweet taste to promote feeding behavior in Drosophila melanogaster. Cell reports. 2019; 27(6):1675-85. https://doi.org/10.1016/j.celrep.2019.04.027 PMID: 31067455

54. Ezenwa VO, Gerardo NM, Inouye DW, Medina M, Xavier JB. Animal behavior and the microbiome. Science. 2012; 338(6104):198-9. https://doi.org/10.1126/science.1227412 PMID: 23066064

55. Provensi G, Schmidt SD, Boehme M, Bastiaanssen TF, Rani B, Costa A, et al. Preventing adolescent stress-induced cognitive and microbiome changes by diet. PNAS. 2019; 116(19):9644-51. https://doi. org/10.1073/pnas.1820832116 PMID: 31010921

56. Desbonnet L, Clarke G, Shanahan F, Dinan TG, Cryan JF. Microbiota is essential for social development in the mouse. Molecular psychiatry. 2014; 19(2):146-8. https://doi.org/10.1038/mp.2013.65 PMID: 23689536

57. Ringelberg J. The photobehaviour of Daphnia spp. as a model to explain diel vertical migration in zooplankton. Biological Reviews. 1999; 74(4):397-423.

58. Cooper RO, Cressler CE. Characterization of key bacterial species in the Daphnia magna microbiota using shotgun metagenomics. Scientific reports. 2020; 10(1):1-1. 